Research Paper

\title{
Prevalence of and risk factors for presenting initial respiratory symptoms in patients undergoing surgery for lung cancer
}

\author{
Xiang-Lin $\mathrm{Hu}^{1 *}$, Song-Tao $\mathrm{Xu}^{2 *}$, Xiao-Cen Wang1, Dong-Ni Hou${ }^{1}$, Cui-Cui Chen ${ }^{1}$, Yuan-Lin Song1, Dong \\ Yang $^{1 凶}$ \\ 1. Department of Pulmonary Medicine, Zhongshan Hospital, Fudan University, Shanghai, China \\ 2. Department of Thoracic Surgery, Zhongshan Hospital, Fudan University, Shanghai, China \\ *These authors contributed equally to this work. \\ $\triangle$ Corresponding author: Dong Yang, Department of Pulmonary Medicine, Zhongshan Hospital, Fudan University, 180 Fenglin Road, Shanghai 200032, China. \\ Email: yang.dong@zs-hospital.sh.cn; Tel: +86-21-64041990-2445 \\ (C) Ivyspring International Publisher. This is an open access article distributed under the terms of the Creative Commons Attribution (CC BY-NC) license \\ (https://creativecommons.org/licenses/by-nc/4.0/). See http://ivyspring.com/terms for full terms and conditions.
}

Received: 2018.03.21; Accepted: 2018.07.22; Published: 2018.08.31

\begin{abstract}
Background: Patients with early stage lung cancer seldom present initial respiratory symptoms, causing a delayed diagnosis and missed opportunity to receive operation. This study aimed to investigate the prevalence of initial respiratory symptoms and identity what factors would predispose lung cancer patients to present initial respiratory symptoms in patients undergoing lung cancer surgery.

Methods: A retrospective chart review was conducted on 3,203 patients undergoing surgery for primary lung cancer. The prevalence of initial respiratory symptoms was investigated and the comparisons of clinicopathological parameters were performed between patients with and without initial respiratory symptoms or between patients with single and multiple initial respiratory symptoms. Independent risk factors for presenting initial respiratory symptoms or multiple initial respiratory symptoms were identified using a logistic regression.

Results: A total of $1,474(46.0 \%)$ patients with lung cancer were admitted to hospital due to present initial respiratory symptoms. Symptom clusters of cough or sputum (33.1\%) and bloody sputum or hemoptysis (16.7\%) presented as the two major chief complaints for medical consultation while chest pain (6.9\%) and chest distress or dyspnea (5.6\%) remained relatively unusual. Multiple analyses found that coexisting chronic obstructive pulmonary disease $(\mathrm{OR}=1.70,95 \% \mathrm{Cl}=1.41-2.05)$, tumor size $>3 \mathrm{~cm}(\mathrm{OR}=2.27,95 \%$ $\mathrm{Cl}=1.93-2.67)$, squamous cell carcinoma $(\mathrm{OR}=2.22,95 \% \mathrm{Cl}=1.86-2.65)$, tumor located in left lower lung $(\mathrm{OR}=1.39,95 \% \mathrm{Cl}=1.10-1.74)$ and advanced tumor stage $(\mathrm{OR}=1.27,95 \% \mathrm{Cl}=1.06-1.52)$ were independent risk factors for presenting initial respiratory symptoms. Furthermore, current smoking $(O R=1.36,95 \%$ $\mathrm{Cl}=1.07-1.73)$, tumor size $>3 \mathrm{~cm}(\mathrm{OR}=1.53,95 \% \mathrm{Cl}=1.21-1.93)$ and squamous cell carcinoma $(\mathrm{OR}=1.68$, $95 \% \mathrm{Cl}=1.32-2.15)$ were demonstrated to be independent risk factors for presenting multiple initial respiratory symptoms.

Conclusions: Presenting initial respiratory symptoms was the common cause for medical consultation in patients undergoing lung cancer surgery. Patients with lung cancer in larger tumor size or squamous cell carcinoma more likely presented initial and even multiple initial respiratory symptoms.
\end{abstract}

Key words: lung neoplasm, respiratory symptom, clinicopathological feature, risk factor

\section{Introduction}

Lung cancer is the most frequently diagnosed cancer and serves as the leading cause of cancer-related death in the world, including in China ${ }^{[1,2]}$. In recent years, despite advances in treatment and management, the prognosis of lung cancer patients is still poor with the reported median survival time of only 22.7 months ${ }^{[3]}$. At present, diagnosis of lung caner as early as possible and then 
treatment with curative surgery remain the preferred modality to improve patients' long-term survival[ ${ }^{[4]}$. But regrettably, due to the insidious onset and rapid progression, most lung cancer patients are diagnosed at locally advanced or disseminated stage when a surgical intervention is no longer appropriate ${ }^{[5]}$. Over the last decades, to detect lung cancer at earlier stage and elevate resectability rates, lung cancer screening through low-dose computed tomography (CT) has been proved to be an effective solution and be able to reduce lung cancer mortality ${ }^{[6,7]}$. However, there also exist risks and limitations about screening such as accumulation of radiation, over diagnosis on indolent tumors that otherwise may not cause clinical symptoms, and importantly, inequitable screening resources and thus difficulty to coverage for all high-risk individuals ${ }^{[8,9]}$.

Hence, in the meantime, much attention has also been paid to symptoms, especially initial respiratory symptoms, that may indicate the incidence of lung cancer ${ }^{[10-12]}$. It was suggested that many patients with lung cancer had subtle shifts in symptoms when they were diagnosed and the majority of them were actually caused by the cancer ${ }^{[13,14]}$. Several studies have consistently demonstrated that presence of hemoptysis is well associated with lung cancer diagnosis and acts as the strongest symptom predictor of lung cancer in those high-risk population ${ }^{[15,16]}$. In fact, although the other respiratory symptoms such as cough, sputum, chest distress or chest pain are relatively less specific in indicating lung cancer, nowadays, as the increasing morbidity of lung cancer, recognizing initial respiratory symptoms as warnings of lung cancer in individuals who are at an increased risk for lung carcinogenesis has been highly concerned by today clinicians. Therefore, it is of great importance to figure out what kinds of patients with lung cancer are more susceptible to initial respiratory symptoms for medical consultation and what kinds of patients with lung cancer are not. To our knowledge, this is the first study designed to identity independent risk factors for presenting initial respiratory symptoms among patients undergoing lung cancer surgery.

\section{Methods}

\section{Patients}

During the period January 2008 to April 2014, consecutive patients undergoing thoracic surgery for primary lung cancer in the department of thoracic surgery of Zhongshan Hospital of Fudan University were retrospectively reviewed. Demographic and medical information were retrieved from the hospital electronic records. As shown in figure 1. In 3,484 patients with complete information, 209 patients with lung cancer which was not primary but metastasis originating from liver cancer, colorectal cancer or other cancers were excluded, so were 72 patients visiting for a second operation because of postoperative lung cancer recurrence after the previous surgery. Ultimately, a total of 3,203 patients were retrospectively analyzed.

\section{Clinicopathological parameters}

In the present study, the initial respiratory symptoms due to the incidence of lung cancer were

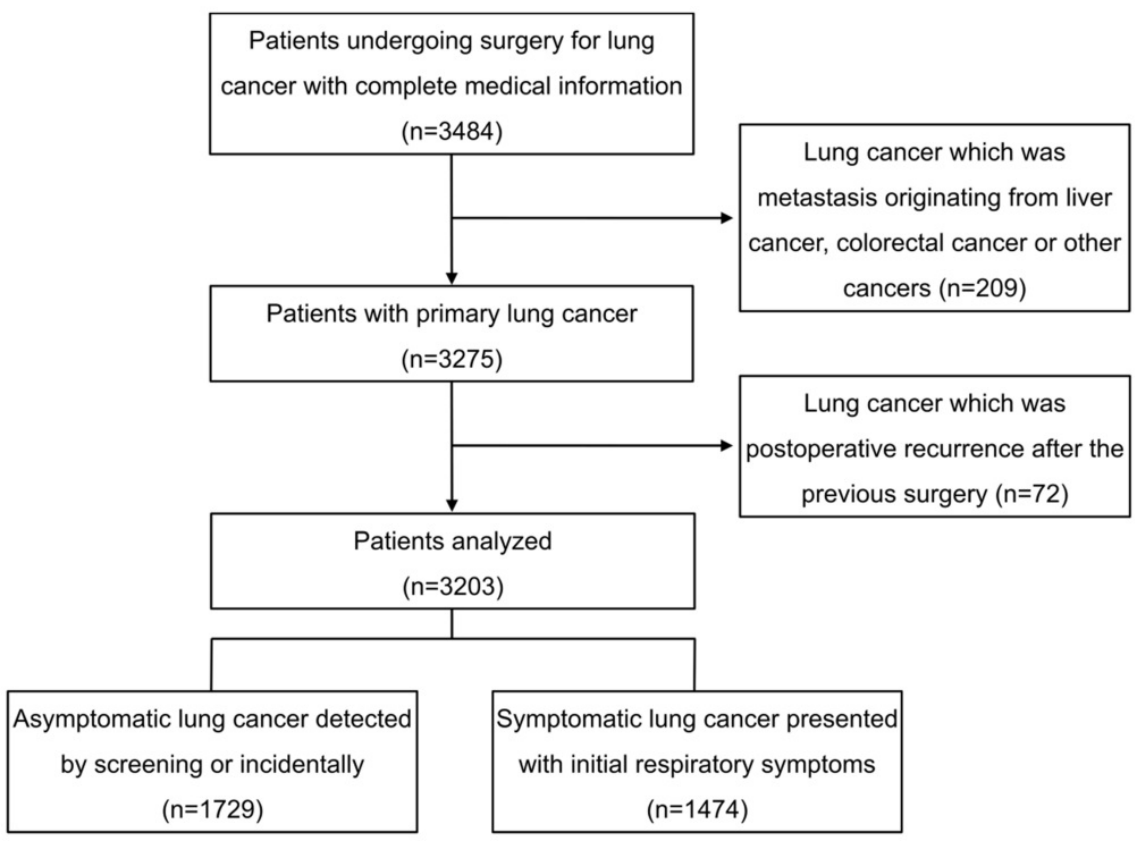

Figure 1. Flow chart showing patients inclusion for this analysis. 
mainly grouped into four clusters: cough or sputum, bloody sputum or hemoptysis, chest distress or dyspnea and chest pain. Presenting any one of these symptoms was considered symptomatic and several different initial respiratory symptoms may be concurrently presented on the same patient. Asymptomatic lung cancer referred to lung cancer without any initial respiratory symptoms which was detected by screening or incidentally through a routine health examination or other occasional pathways based on CT or chest radiography. Patients concurrently presenting two or more than two different clusters of initial respiratory symptoms were considered multi-symptomatic while patients presenting with only one cluster of initial respiratory symptoms were considered mono-symptomatic.

Patients' spirometric lung functions such as forced expiratory volume in one second $\left(\mathrm{FEV}_{1}\right)$, ratio of $\mathrm{FEV}_{1}$ to predicted values ( $\mathrm{FEV}_{1} \%$ pred), forced vital capacity (FVC), ratio of FVC to predicted values (FVC\% pred), diffusion capacity for carbon monoxide (DLCO) and ratio of residual volume to total lung capacity (RV/TLC) were collected and a post-bronchodilator $\mathrm{FEV}_{1} / \mathrm{FVC}<70 \%$ was defined as chronic obstructive pulmonary disease (COPD) ${ }^{[17]}$. Demographic and clinicopathological parameters including age, gender, smoking status, comorbidity of COPD, tumor size (the largest diameter of lung cancer), tumor location, histological subtype, histological differentiation and pathological TNM stage were collected for comparative analyses between patients with and without initial respiratory symptoms or between patients with single and multiple initial respiratory symptoms.

\section{Statistical analysis}

Continuous data were shown as mean \pm standard deviation or median with interquartile range and compared using Student's t-test or the nonparametric Mann-Whitney $\mathrm{U}$ test as appropriate. Categorical data were shown as frequencies with percentages and compared using chi-square test. Ranked data such as tumor stage were compared using the Mann-Whitney $U$ test. The parameters with $P<0.10$ in univariate analysis were entered into a stepwise multivariate binary logistic regression analysis to identify independent risk factors for presenting initial respiratory symptoms or presenting multiple initial respiratory symptoms. Odds ratio (OR) with 95\% confidence interval (CI) was used as a measure of the magnitude of association. All statistical analyses were conducted using SPSS for Windows (Version 17.0, Chicago, IL, USA). A two-sided $P$ value $<0.05$ was considered statistically significant for all analyses.

\section{Results}

\section{Prevalence of initial respiratory symptoms}

Among the 3,203 patients with surgically treated lung cancer, $1,474(46.0 \%)$ patients were admitted to department of thoracic surgery due to present initial respiratory symptoms while the other 1,729 (54.0\%) patients were asymptomatic and admitted due to detection of lung cancer by screening or accident. Expressed as median with interquartile range, the symptom lead time, time from initiation of respiratory symptoms to admission, was $1.0(0.5-3.0)$ month, which was significantly longer than the time from detection of lung cancer to admission with 0.7 $(0.3-2.0)$ months $(P<0.05)$.

As shown in figure 2. Among the 1,474 (46.0\%) patients with initial respiratory symptoms, approximately two thirds of patients $(30.6 \%)$ were mono-symptomatic $(n=979)$ while merely one third of patients $(15.5 \%)$ were multi-symptomatic $(n=495)$. The 495 patients with multiple symptoms were consisted of 471 patients with double symptoms, 22 patients with triple symptoms and 2 patients with quadruple symptoms. Cough or sputum ranked as the most common chief complaint with a prevalence of $33.1 \%$, followed by bloody sputum or hemoptysis with $16.7 \%$, chest pain and chest distress or dyspnea accounted for the similarly lower prevalence with $6.9 \%$ and 5.6\%, respectively. Among the multi-symptomatic patients, cough or sputum combined with bloody sputum or hemoptysis was the most common form of presenting multiple initial respiratory symptoms with the presence in 332 patients.

As shown in table 1. Except for FVC, spirometric parameters including $\mathrm{FEV}_{1}, \mathrm{FEV}_{1} \%$ pred, $\mathrm{FVC} \%$ pred, $\mathrm{D}_{\mathrm{LCO}}$ and RV/TLC of patients with symptomatic lung cancer were all inferior to their counterparts of patients with asymptomatic lung cancer $(P<0.05)$, indicating that lung cancer patients presenting initial respiratory symptoms tended to have worse pulmonary ventilation and gas exchange function as well as higher gas retention. However, within the comparisons of lung functions between patients with single and multiple initial respiratory symptoms, only $\mathrm{FEV}_{1} \%$ pred was found significantly decreased in the patients presenting multiple initial respiratory symptoms $(P<0.05)$.

\section{Risk factors for presenting initial respiratory symptoms}

As shown in table 2, clinicopathological parameters possibly responsible for presenting initial respiratory symptoms were compared between patients with asymptomatic and symptomatic lung 
cancer. Compared to patients with asymptomatic lung cancer, patients with symptomatic lung cancer were inclined to be males $(67.4 \%$ vs $55.3 \%)$, current smokers (32.4\% vs $22.8 \%$ ) and with coexisting COPD (28.4\% vs $15.2 \%)$. Tumors in size larger than $3 \mathrm{~cm}$ (56.2\% vs $29.3 \%)$, located in left lower lung $(17.8 \%$ vs $14.1 \%)$, of squamous cell carcinoma type (41.4\% vs $18.6 \%)$ or in poor differentiation ( $50.0 \%$ vs $43.4 \%$ ) were more frequently found in patients with symptomatic lung cancer than in patients with asymptomatic lung cancer. Moreover, lung cancer patients presenting initial respiratory symptoms had more possibility at stage II $(16.8 \%$ vs $11.5 \%)$ or stage III/IV (29.1\% vs $21.9 \%$ ) than lung cancer patients without initial respiratory symptoms.

As shown in table 3, multiple analyses demonstrated that comorbidity of COPD (OR=1.70, $95 \% \mathrm{CI}=1.41-2.05)$, tumor size $>3 \mathrm{~cm}(\mathrm{OR}=2.27,95 \%$ $\mathrm{CI}=1.93-2.67)$, tumor location in left lower lung $(\mathrm{OR}=1.39, \quad 95 \% \quad \mathrm{CI}=1.10-1.74)$, squamous cell carcinoma $\quad(\mathrm{OR}=2.22, \quad 95 \% \quad \mathrm{CI}=1.86-2.65) \quad$ and advanced tumor stage $(\mathrm{OR}=1.27,95 \% \mathrm{CI}=1.06-1.52)$ were independent risk factors for presenting initial respiratory symptoms.

A

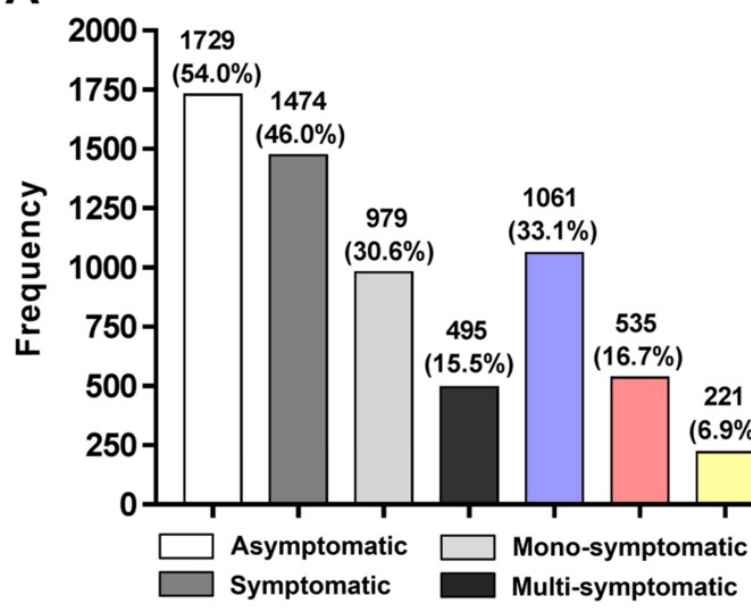

\section{Risk factors for presenting multiple initial respiratory symptoms}

As shown in table 2, significant differences of clinicopathological parameters can be continually found between patients with mono-symptomatic lung cancer and multi-symptomatic lung cancer. Males ( $74.9 \%$ vs $63.6 \%)$, current smokers $(40.0 \%$ vs $28.6 \%)$ and comorbidity of COPD (31.7\% vs $26.8 \%)$ were further increased in patients with multi-symptomatic lung cancer versus patients with mono-symptomatic lung cancer. Tumors in size larger than $3 \mathrm{~cm}$ were more frequently found in multi-symptomatic lung cancer patients than in mono-symptomatic lung cancer patients $(65.9 \%$ vs $51.4 \%)$. The proportion of squamous cell carcinoma was significantly higher in multi-symptomatic lung cancer patients versus mono-symptomatic lung cancer patients $(52.5 \%$ vs $35.8 \%$ ). Despite adenocarcinoma is generally regarded as the most common histological subtype of lung cancer ${ }^{[18]}$, our study demonstrated that, the proportion of squamous cell carcinoma exceeded adenocarcinoma ( $52.5 \%$ vs $41.0 \%$ ) to be the majority in multi-symptomatic lung cancer patients.

B

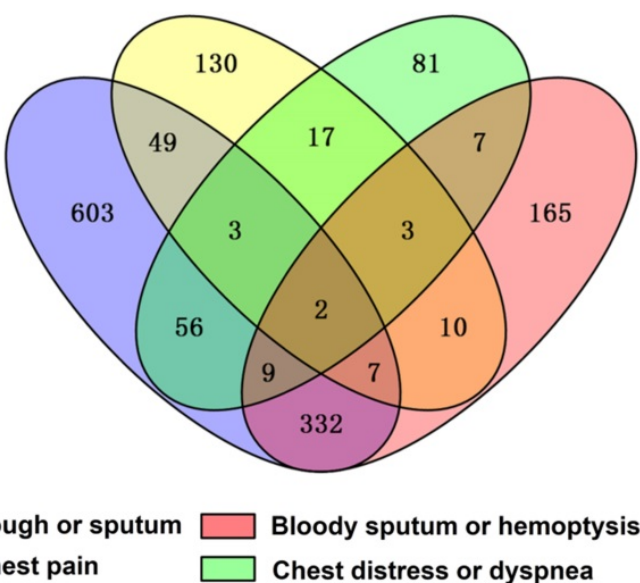

Figure 2. Chart of frequency distribution (A) and Venn diagram (B) showing numbers of each cluster of initial respiratory symptoms. Patients with asymptomatic lung cancer $(n=1,729)$ were slightly more than patients with symptomatic lung cancer $(n=1,474)$. And among the $1,474(46.0 \%)$ patients with symptomatic lung cancer, up to 979 (30.6\%) patients presented only one cluster of initial respiratory symptoms while merely 495 (15.5\%) patients concurrently presented two or more than two different clusters of initial respiratory symptoms. Cough or sputum $(n=1061)$ and bloody sputum or hemoptysis $(n=535)$ served as the major two chief complaints while chest pain $(n=221)$ and chest distress or dyspnea $(n=178)$ remained relatively unusual in the causes of admission to hospital for lung cancer surgery.

Table 1. Comparisons of lung functions between patients with and without initial respiratory symptoms or between patients with single and multiple initial respiratory symptoms.

\begin{tabular}{llllll}
\hline Lung function & Asymptomatic $(\mathrm{n}=1729)$ & Symptomatic $(\mathrm{n}=1474)$ & $P$-value & Mono-symptomatic $(\mathrm{n}=979)$ & Multi-symptomatic $(\mathrm{n}=495)$ \\
\hline FEV 1 (L) & $2.39 \pm 0.63$ & $2.27 \pm 0.61$ & $<0.001^{*}$ & $2.27 \pm 0.61$ & $2.28 \pm 0.60$ \\
FEV $1 \%$ pred(\%) & $90.46 \pm 18.22$ & $83.87 \pm 18.52$ & $<0.001^{*}$ & $84.79 \pm 18.40$ & $82.07 \pm 18.65$ \\
FVC(L) & $3.09 \pm 0.75$ & $3.06 \pm 0.73$ & 0.267 & $3.03 \pm 0.74$ & $3.11 \pm 0.71$ \\
FVC\%pred(\%) & $91.80 \pm 15.12$ & $88.94 \pm 15.33$ & $<0.001^{*}$ & $89.26 \pm 15.20$ & $0.008^{*}$ \\
DLCO & $18.79 \pm 5.39$ & $18.02 \pm 5.52$ & $<0.001^{*}$ & $17.93 \pm 5.26$ & 0.05 \\
RV/TLC(\%) & $37.27 \pm 7.83$ & $37.98 \pm 8.02$ & $0.011^{*}$ & $37.94 \pm 8.09$ & 0.268 \\
\hline
\end{tabular}

FEV1: Forced expiratory volume in 1 second; FEV1\% pred: Ratio of FEV1 to predicted values; FVC: Forced vital capacity; FVC \% pred: Ratio of FVC to predicted values; RV/TLC: Ratio of residual volume to total lung capacity; DLCO: Diffusion capacity for carbon monoxide. ${ }^{*} P<0.05$ 
Table 2. Univariate analyses of risk factors associated with presenting initial respiratory symptoms or presenting multiple initial respiratory symptoms.

\begin{tabular}{|c|c|c|c|c|c|c|}
\hline Parameter & Asymptomatic $(\mathrm{n}=1729)$ & Symptomatic $(n=1474)$ & $P$-value & Mono-symptomatic $(n=979)$ & Multi-symptomatic $(\mathrm{n}=495)$ & $P$-value \\
\hline Age(years) & $60.3 \pm 9.8$ & $60.7 \pm 9.8$ & 0.264 & $60.5 \pm 9.9$ & $61.1 \pm 9.6$ & 0.300 \\
\hline Gender & & & $<0.001^{*}$ & & & $<0.001^{*}$ \\
\hline Female & $773(44.7 \%)$ & $480(32.6 \%)$ & & $356(36.4 \%)$ & $124(25.1 \%)$ & \\
\hline Male & $956(55.3 \%)$ & $994(67.4 \%)$ & & $623(63.6 \%)$ & $371(74.9 \%)$ & \\
\hline Smoking status & & & $<0.001^{*}$ & & & $<0.001^{*}$ \\
\hline Former or Never & $1335(77.2 \%)$ & $996(67.6 \%)$ & & $699(71.4 \%)$ & $297(60.0 \%)$ & \\
\hline Current & $394(22.8 \%)$ & $478(32.4 \%)$ & & $280(28.6 \%)$ & $198(40.0 \%)$ & \\
\hline Comorbidity of COPD & & & $<0.001^{*}$ & & & $0.046^{*}$ \\
\hline No & $1466(84.8 \%)$ & $1055(71.6 \%)$ & & $717(73.2 \%)$ & $338(68.3 \%)$ & \\
\hline Yes & $263(15.2 \%)$ & $419(28.4 \%)$ & & $262(26.8 \%)$ & $157(31.7 \%)$ & \\
\hline Tumor size(cm) & & & $<0.001^{*}$ & & & $<0.001^{*}$ \\
\hline$\leq 3$ & $1223(70.7 \%)$ & $645(43.8 \%)$ & & $476(48.6 \%)$ & $169(34.1 \%)$ & \\
\hline$>3$ & $506(29.3 \%)$ & $829(56.2 \%)$ & & $503(51.4 \%)$ & $326(65.9 \%)$ & \\
\hline Tumor location & & & $0.003^{*}$ & & & 0.366 \\
\hline Right upper lung & $579(33.5 \%)$ & $418(28.4 \%)$ & & $274(28.0 \%)$ & $144(29.1 \%)$ & \\
\hline Right middle lung & $140(8.1 \%)$ & $133(9.0 \%)$ & & $91(9.3 \%)$ & $42(8.5 \%)$ & \\
\hline Right lower lung & $309(17.9 \%)$ & $285(19.3 \%)$ & & $201(20.5 \%)$ & $84(17.0 \%)$ & \\
\hline Left upper lung & $457(26.4 \%)$ & $375(25.4 \%)$ & & $237(24.2 \%)$ & $138(27.9 \%)$ & \\
\hline Left lower lung & $244(14.1 \%)$ & $263(17.8 \%)$ & & $176(18.0 \%)$ & $87(17.6 \%)$ & \\
\hline Histological subtype & & & $<0.001^{*}$ & & & $<0.001^{*}$ \\
\hline Adenocarcinoma & $1311(75.8 \%)$ & $766(52.0 \%)$ & & $563(57.5 \%)$ & $203(41.0 \%)$ & \\
\hline Squamous cell & $322(18.6 \%)$ & $610(41.4 \%)$ & & $350(35.8 \%)$ & $260(52.5 \%)$ & \\
\hline Small cell & $25(1.4 \%)$ & $32(2.2 \%)$ & & $20(2.0 \%)$ & $12(2.4 \%)$ & \\
\hline Others & $71(4.1 \%)$ & $66(4.5 \%)$ & & $46(4.7 \%)$ & $20(4.0 \%)$ & \\
\hline Histological differentiation & & & $<0.001^{*}$ & & & 0.544 \\
\hline Well or Moderate & $978(56.6 \%)$ & $737(50.0 \%)$ & & $495(50.6 \%)$ & $242(48.9 \%)$ & \\
\hline Poor & $751(43.4 \%)$ & $737(50.0 \%)$ & & $484(49.4 \%)$ & $253(51.1 \%)$ & \\
\hline Pathological TNM stage & & & $<0.001^{*}$ & & & 0.324 \\
\hline 0 or I & $1152(66.6 \%)$ & $797(54.1 \%)$ & & $538(55.0 \%)$ & $259(52.3 \%)$ & \\
\hline II & $199(11.5 \%)$ & $248(16.8 \%)$ & & $163(16.6 \%)$ & $85(17.2 \%)$ & \\
\hline III or IV & $378(21.9 \%)$ & $429(29.1 \%)$ & & $278(28.4 \%)$ & $151(30.5 \%)$ & \\
\hline
\end{tabular}

COPD: Chronic obstructive pulmonary disease. ${ }^{*} P<0.05$

Table 3. Multivariate analyses of independent risk factors for presenting initial respiratory symptoms

\begin{tabular}{llll}
\hline Parameter & Odds ratio & $95 \%$ Confidence interval & $P$-value \\
\hline Comorbidity of COPD & & & \\
No & 1.00 & & $<0.001^{*}$ \\
Yes & 1.70 & $1.41-2.05$ & \\
Tumor size(cm) & & & \\
$\leq 3$ & 1.00 & & $<0.001^{*}$ \\
$>3$ & 2.27 & $1.93-2.67$ & $0.008^{*}$ \\
Tumor location & & & \\
Right upper lung & 1.00 & & $0.011^{*}$ \\
Right middle lung & 1.45 & $1.09-1.93$ & $0.022^{*}$ \\
Right lower lung & 1.29 & $1.04-1.60$ & 0.483 \\
Left upper lung & 1.07 & $0.88-1.31$ & $0.005^{*}$ \\
Left lower lung & 1.39 & $1.10-1.74$ & $<0.001^{*}$ \\
Histological subtype & & & $<0.001^{*}$ \\
Adenocarcinoma & 1.00 & & 0.073 \\
Squamous cell & 2.22 & $1.86-2.65$ & $0.023^{*}$ \\
Small cell & 1.66 & $0.95-2.87$ & $0.018^{*}$ \\
Others & 1.52 & $1.06-2.18$ & \\
Pathological TNM stage & & & 0.064 \\
0 or I & 1.00 & & $0.011^{*}$ \\
II & 1.24 & $0.99-1.55$ & \\
III or IV & 1.27 & $1.06-1.52$ &
\end{tabular}

COPD: Chronic obstructive pulmonary disease. ${ }^{*} P<0.05$

As shown in table 4, multiple analyses found that only tumors in size $>3 \mathrm{~cm} \quad(\mathrm{OR}=1.53,95 \%$ $\mathrm{CI}=1.21-1.93)$ and squamous cell carcinoma $(\mathrm{OR}=1.68,95 \% \mathrm{CI}=1.32-2.15)$ continued to be significant risk factors for presenting multiple initial respiratory symptoms. Interestingly, although current smoking was not an independent risk factor for presenting initial respiratory symptoms, it was an independent risk factor for presenting multiple initial respiratory symptoms in patients with initial respiratory symptoms $(\mathrm{OR}=1.36,95 \% \mathrm{CI}=1.07-1.73)$.

Table 4. Multivariate analyses of independent risk factors for presenting multiple initial respiratory symptoms

\begin{tabular}{llll}
\hline Parameter & Odds ratio & $95 \%$ Confidence interval & $P$-value \\
\hline $\begin{array}{l}\text { Smoking status } \\
\text { Former or Never }\end{array}$ & 1.00 & & \\
$\begin{array}{l}\text { Current } \\
\text { Tumor size }(\mathrm{cm})\end{array}$ & 1.36 & $1.07-1.73$ & $0.011^{*}$ \\
$\leq 3$ & & & \\
$>3$ & 1.00 & & $<0.001^{*}$ \\
Histological subtype & 1.53 & $1.21-1.93$ & $0.001^{*}$ \\
$\begin{array}{l}\text { Adenocarcinoma } \\
\text { Squamous cell }\end{array}$ & 1.00 & & \\
Small cell & 1.68 & $1.32-2.15$ & $<0.001^{*}$ \\
Others & 1.46 & $0.69-3.05$ & 0.321 \\
\hline
\end{tabular}

${ }^{*} P<0.05$

\section{Discussion}

To date, although the disparities between patients with symptomatic lung cancer and asymptomatic lung cancer have been observed in several studies, most studies placed asymptomatic lung cancer patients detected by screening or incidentally as the research subjects while treated symptomatic lung cancer patients with initial 
respiratory symptoms as the controls[19-21]. Few studies focused on describing the clinicopathological characteristics of lung cancer patients visiting due to present initial respiratory symptoms. Our study demonstrated that patients presenting initial respiratory symptoms tended to be males, current smokers and with coexisting COPD. Comorbidity of COPD was further proved to be an independent risk factor for presenting initial respiratory symptoms. To some extent, this can be explained by the hypothesis that tumors may be more easily to obstruct the otherwise narrowed airways with hyper-mucus secretion in patients with $\mathrm{COPD}^{[22]}$ and thus cause initial respiratory symptoms early.

In our study, an obvious delay of visiting doctors was found in lung cancer patients presenting initial respiratory symptoms, indicating that patients themselves hardly took respiratory symptoms seriously as much as the positive radiography findings. Their negligence of initial respiratory symptoms may potentially cause the disease progression. Thus, in the future, efforts should be made to educate high-risk individuals to be alert to their initial respiratory symptoms. Furthermore, our study found that lung cancer patients presenting initial respiratory symptoms had worse lung functions. Since enough $\mathrm{FEV}_{1}$ and $\mathrm{D}_{\mathrm{LCO}}$ are necessary for lung resection [23], it can be speculated that patients presenting initial respiratory symptoms with worse lung functions may have a lower probability to adequately tolerate lung cancer surgery.

Our study showed that patients with lung cancer presenting initial respiratory symptoms had more chance with tumors in larger size, located in left lower lung, in squamous cell type, with poor differentiation and at advanced-stage. Our results partially kept highly in line with the study of Orrason and colleagues [24], in which the CT-detected tumors were deemed as the focused subjects, that the CT-detected tumors were smaller, at earlier TNM stages than symptomatic tumors and more often of adenocarcinoma. Unexpectedly, in addition to tumor size and histology which were easily understood to be associated with initial respiratory symptoms of lung cancer, we uncovered tumor location in left lower lung may also play a role in facilitating initial respiratory symptoms. Although this novel finding remained very obscure in pathophysiologic perspective, Study from Kudo and colleagues[25] supported that tumor location in left lower lung did confer certain distinct clinicopathological features of lung cancer.

Strikingly, in this study, we identified that the most two dominant contributors to initial respiratory symptoms went to larger tumor size and histology of squamous cell carcinoma, which can go on playing their facilitating roles in presenting multiple initial respiratory symptoms. Although a previous study found that the proportion of symptoms indicative of lung cancer exerted a well linear relationship with the tumor size ${ }^{[26], ~ t h e ~ c o n c l u s i o n ~ t h a t ~ t u m o r ~ s i z e ~ w a s ~ a n ~}$ independent risk factor for presenting initial respiratory symptoms was not demonstrated until this present study. And noteworthily, evidence suggested that for patients with incidence of lung cancer, the proportion of adenocarcinoma is going up over time[18,27]. Our study demonstrated that lung adenocarcinomas were not prone to cause patients to present initial respiratory symptoms. Thus, an unoptimistic speculation might be, in the future, decreasing proportion of patients with lung cancer would be timely admitted for surgery thanks to the presence of initial respiratory symptoms.

In addition, a very interesting finding of this study was that current smoking was not an independent risk factor for presenting initial respiratory symptoms but an independent risk factor for presenting multiple initial respiratory symptoms. And this phenomenon might be attributed to the following interpretation: as demonstrated in our study, lung cancer patients with or without initial respiratory symptoms were mainly determined by tumor size and histology. Compared to the potent and intrinsic effects shaped by tumor size and histology, the contribution of current smoking might become trivial and negligible. However, when patients already presented initial respiratory symptoms, current smoking would overlay its additive deteriorating effects on initial respiratory symptoms to be multiple initial respiratory symptoms.

Finally, there existed several limitations in this study. First, although the population in this study was very large, all patients were from one institution, indicating the potential for selection bias. Secondly, due to lack of full information, we cannot compare more differences between lung cancer patients with and without initial respiratory symptoms, such as postoperative complications and postoperative survival rates. Thirdly, this was a retrospectively designed study, future prospective studies are supposed to confirm the conclusions of our study.

\section{Conclusions}

In summary, this study performed a single-institution retrospective analysis based on a large Chinese population in patients undergoing lung cancer surgery. Symptom clusters of cough or sputum and bloody sputum or hemoptysis were the two most common initial respiratory symptoms causing to medical consultation. Patients with lung cancer in 
larger tumor size or histology of squamous cell carcinoma were more susceptible to initial respiratory symptoms and even multiple initial respiratory symptoms.

\section{Abbreviations}

COPD: Chronic obstructive pulmonary disease; CT: Computed tomography; $\mathrm{FEV}_{1}$ : Forced expiratory volume in 1 second; $\mathrm{FEV}_{1} \%$ pred: Ratio of $\mathrm{FEV}_{1}$ to predicted values; FVC: Forced vital capacity; FVC\% pred: Ratio of FVC to predicted values; RV/TLC: Ratio of residual volume to total lung capacity; DLCo: Diffusion capacity for carbon monoxide; OR: Odds ratio; CI: Confidence interval.

\section{Acknowledgments}

We should give our thanks to the departments of medical record and lung function to offer data records.

\section{Ethics approval and consent to participate}

This study was approved by the ethics committee of Zhongshan Hospital, Fudan University.

\section{Funding}

This work was supported by State's Key Project of Research and Development Plan (2017YFC1310602, 2017YFC1310600), Research Foundation of Shanghai Municipal Commission of Health and Family Planning (201540078), National Natural Science Foundation of China (81100048, 81500026).

\section{Authors' contributions}

$\mathrm{Hu}$ X-L contributed to the study conception and design, data analysis, interpretation of the data and drafting the manuscript. Yang D, Song Y-L and Xu S-T contributed to the study design, interpretation of the data and critical revision of the manuscript. $\mathrm{Hu} \mathrm{X}-\mathrm{L}$, $\mathrm{Xu}$ S-T, Wang X-C, Hou D-N and Chen C-C contributed to the acquisition of the data. All authors read and approved the final manuscript.

\section{Competing Interests}

The authors have declared that no competing interest exists.

\section{References}

[1] Malhotra J, Malvezzi M, Negri E, et al. Risk factors for lung cancer worldwide. Eur Respir J. 2016;48(3):889-902.

[2] Chen W, Zheng R, Baade PD, et al. Cancer statistics in China, 2015. CA Cancer J Clin. 2016;66(2):115-32.

[3] Fan H, Shao ZY, Xiao YY, et al. Incidence and survival of non-small cell lung cancer in Shanghai: a population-based cohort study. BMJ Open. 2015;5(12):e009419.

[4] Howington JA, Blum MG, Chang AC, et al. Treatment of stage I and II non-small cell lung cancer: Diagnosis and management of lung cancer, 3rd ed: American College of Chest Physicians evidence-based clinical practice guidelines. Chest 2013;143:e278S-e313S.

[5] Wang BY, Huang JY, Cheng CY, et al. Lung cancer and prognosis in taiwan: a population-based cancer registry. J Thorac Oncol. 2013;8(9):1128-35.
[6] Edwards JP, Datta I, Hunt JD, et al. The impact of computed tomographic screening for lung cancer on the thoracic surgery workforce. Ann Thorac Surg. 2014;98(2):447-52.

[7] National Lung Screening Trial Research Team, Aberle DR, Adams AM, et al. Reduced lung-cancer mortality with low-dose computed tomographic screening. N Engl J Med. 2011;365(5):395-409.

[8] Patz EF Jr, Pinsky P, Gatsonis C, et al. Overdiagnosis in low-dose computed tomography screening for lung cancer. JAMA Intern Med. 2014;174(2):269-74.

[9] Eberth JM, Qiu R, Adams SA, et al. Lung cancer screening using low-dose CT: the current national landscape. Lung Cancer. 2014;85(3):379-84.

[10] Taiwo EO, Yorio JT, Yan J, et al. How have we diagnosed early-stage lung cancer without radiographic screening? A contemporary single-center experience. PLoS One. 2012;7(12):e52313.

[11] Crane M, Scott N, O'Hara BJ, et al. Knowledge of the signs and symptoms and risk factors of lung cancer in Australia: mixed methods study. BMC Public Health. 2016;16:508.

[12] Ades AE, Biswas M, Welton NJ, et al. Symptom lead time distribution in lung cancer: natural history and prospects for early diagnosis. Int J Epidemiol. 2014;43(6):1865-73

[13] Gonzalez-Barcala FJ, Falagan JA, Garcia-Prim JM, et al. Symptoms and reason for a medical visit in lung cancer patients. Acta Med Port. 2014;27(3):318-24.

[14] Biswas M, Ades AE, Hamilton W. Symptom lead times in lung and colorectal cancers: what are the benefits of symptom-based approaches to early diagnosis?. Br J Cancer. 2015;112(2):271-7.

[15] Shim J, Brindle L, Simon M, et al. A systematic review of symptomatic diagnosis of lung cancer. Fam Pract. 2014;31(2):137-48.

[16] Walter FM, Rubin G, Bankhead C, et al. Symptoms and other factors associated with time to diagnosis and stage of lung cancer: a prospective cohort study. Br J Cancer. 2015; 112 Suppl 1:S6-13.

[17] Vogelmeier CF, Criner GJ, Martinez FJ, et al. Global Strategy for the Diagnosis, Management, and Prevention of Chronic Obstructive Lung Disease 2017 Report: GOLD Executive Summary. Am J Respir Crit Care Med 2017;195:557-82.

[18] Nakamura H, Saji H. Worldwide trend of increasing primary adenocarcinoma of the lung. Surg Today. 2014;44(6):1004-12.

[19] In KH, Kwon YS, Oh IJ, et al. Lung cancer patients who are asymptomatic at diagnosis show favorable prognosis: a korean Lung Cancer Registry Study. Lung Cancer. 2009;64(2):232-7.

[20] Kocher F, Lunger F, Seeber A, et al. Incidental Diagnosis of Asymptomatic Non-Small-Cell Lung Cancer: A Registry-Based Analysis. Clin Lung Cancer. 2016;17(1):62-7.

[21] Quadrelli S, Lyons G, Colt H, et al. Clinical characteristics and prognosis of incidentally detected lung cancers. Int J Surg Oncol. 2015:287604.

[22] Chillappagari S, Preuss J, Licht S, et al. Altered protease and antiprotease balance during a COPD exacerbation contributes to mucus obstruction. Respir Res. 2015;16:85.

[23] Brunelli A, Kim AW, Berger KI, et al. Physiologic evaluation of the patient with lung cancer being considered for resectional surgery: Diagnosis and management of lung cancer, 3rd ed: American College of Chest Physicians evidence-based clinical practice guidelines. Chest. 2013;143(5 Suppl):e166S-e190S.

[24] Orrason AW, Sigurdsson MI, Baldvinsson K, et al. Incidental detection by computed tomography is an independent prognostic factor for survival in patients operated for nonsmall cell lung carcinoma. ERJ Open Res. 2017;3:2.

[25] Kudo $Y$, Saji $H$, Shimada $Y$, et al. Do tumours located in the left lower lobe have worse outcomes in lymph node-positive non-small cell lung cancer than tumours in other lobes?. Eur J Cardiothorac Surg. 2012;42(3):414-9.

[26] Chen X, Gorlov IP, Ying J, et al. Initial medical attention on patients with early-stage non-small cell lung cancer. PLoS One. 2012;7(3):e32644.

[27] Khakwani A, Rich AL, Powell HA, et al. Lung cancer survival in England: trends in non-small-cell lung cancer survival over the duration of the National Lung Cancer Audit. Br J Cancer. 2013;109(8):2058-65. 